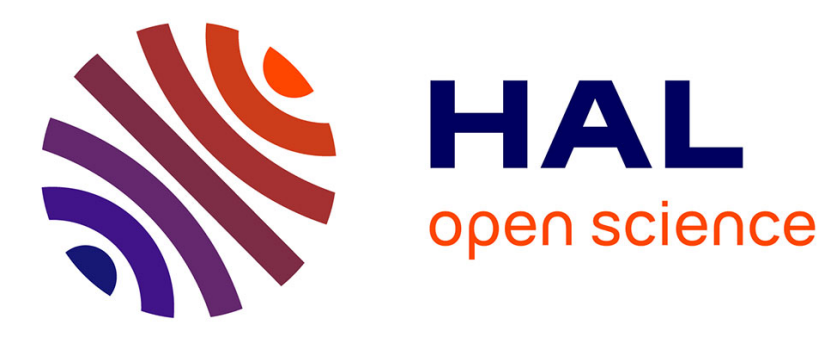

\title{
Impact Cratering and Break up of the Small Bodies of the Solar System
}

J. Leliwa-Kopystyński, M.J. Burchell, D. Lowen

\section{To cite this version:}

J. Leliwa-Kopystyński, M.J. Burchell, D. Lowen. Impact Cratering and Break up of the Small Bodies of the Solar System. Icarus, 2009, 195 (2), pp.817. 10.1016/j.icarus.2008.02.010 . hal-00558515

\section{HAL Id: hal-00558515 https://hal.science/hal-00558515}

Submitted on 22 Jan 2011

HAL is a multi-disciplinary open access archive for the deposit and dissemination of scientific research documents, whether they are published or not. The documents may come from teaching and research institutions in France or abroad, or from public or private research centers.
L'archive ouverte pluridisciplinaire HAL, est destinée au dépôt et à la diffusion de documents scientifiques de niveau recherche, publiés ou non, émanant des établissements d'enseignement et de recherche français ou étrangers, des laboratoires publics ou privés. 


\section{Accepted Manuscript}

Impact Cratering and Break up of the Small Bodies of the Solar System

J. Leliwa-Kopystyński, M.J. Burchell, D. Lowen

PII:

S0019-1035(08)00082-1

DOI: $\quad$ 10.1016/j.icarus.2008.02.010

Reference: $\quad$ YICAR 8613

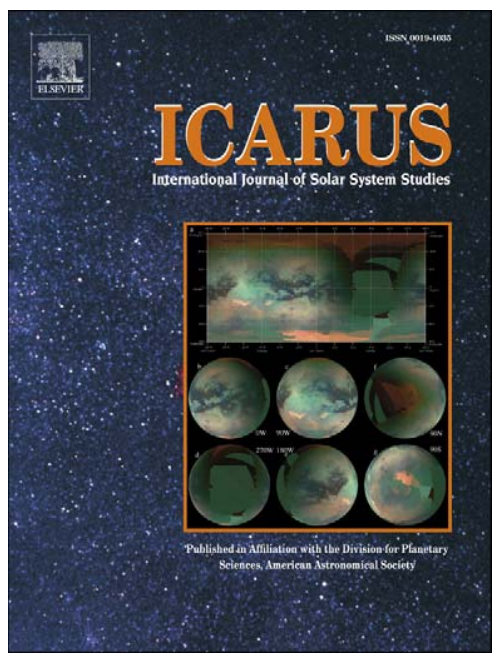

To appear in: Icarus

Received date: 16 November 2006

Revised date: 8 February 2008

Accepted date: 11 February 2008

Please cite this article as: J. Leliwa-Kopystyński, M.J. Burchell, D. Lowen, Impact Cratering and Break up of the Small Bodies of the Solar System, Icarus (2008), doi: 10.1016/j.icarus.2008.02.010

This is a PDF file of an unedited manuscript that has been accepted for publication. As a service to our customers we are providing this early version of the manuscript. The manuscript will undergo copyediting, typesetting, and review of the resulting proof before it is published in its final form. Please note that during the production process errors may be discovered which could affect the content, and all legal disclaimers that apply to the journal pertain. 
Impact Cratering and Break up of the Small Bodies of the Solar System

\author{
J. Leliwa-Kopystyński, ${ }^{\mathrm{a}}$ M. J. Burchell, ${ }^{\mathrm{b}}{ }^{\mathrm{a}}$ and D. Lowen ${ }^{\mathrm{b}}$ \\ ${ }^{a}$ Warsaw University, Institute of Geophysics, Pasteura 7, 02-093 Warszawa, and \\ Space Research Centre of Polish Academy of Sciences, Bartycka 18A, 00-716 Warszawa, \\ Poland \\ ${ }^{\mathrm{b}}$ Centre for Astrophysics and Planetary Sciences, University of Kent \\ Canterbury, Kent, CT2 7NH, UK \\ * Corresponding Author E-mail: M.J.Burchell@kent.ac.uk
}

Number of manuscript pages (excluding figures and tables): 25

Number of figures: 6

Number of tables: 4 
Proposed running header: Impact disruption of icy bodies

Editorial correspondence to:

Dr. M. J. Burchell

Centre for Astrophysics and Planetary Sciences, University of Kent Canterbury, Kent, CT2 7NH, UK

E-mail: M.J.Burchell@kent.ac.uk Fax: 44 (0)1227 827558, Tel: +44 (0)1227 823321 


\begin{abstract}
We consider the largest impact craters observed on small satellites and asteroids and the impact disruption of such bodies. Observational data are considered from 21 impact-like structures on 13 satellites and 8 asteroids (target body radii in the range $0.7-265 \mathrm{~km}$ ). If the radius of the target body is $R$ and the diameter of the largest crater observed on this body $D$, the ratio $D / R$ is then the main observational parameter of interest. This is found on the observed bodies and compared to data obtained in the laboratory. Taking the largest observed value for $D / R$ as a proxy for the ratio $D_{c} / R$ (where $D_{\mathrm{c}}$ is the diameter of the largest crater that can be formed on a body without shattering it) it was found that for the observed icy satellites $D_{c, \text { icy }} \approx 1.2 R$ and for the asteroids and the rocky satellites $D_{c, \text { rocky }} \approx 1.6 R$. In laboratory experiments with ice targets at impactor speeds of 1 to $3 \mathrm{~km} \mathrm{~s}^{-1}$ we obtained $D_{c, \text { icy }} \approx 1.64 R$.
\end{abstract}

Keywords: Asteroids; Collisional physics; Impact processes; Surfaces, asteroids; Surfaces, satellites 


\section{List of symbols.}

\section{Unless otherwise stated, SI units are used throughout this paper.}

$D \quad$ Diameter of the largest crater identified on the target [m]

$D_{c} \quad$ Diameter of the largest (critical) crater that can be formed on the target [m]

$E_{\mathrm{c}} \quad$ Impactor energy threshold for catastrophic break up of the target [J]

$G \quad$ Gravity constant, $6.672 \times 10^{-11}\left[\mathrm{~m}^{3} \mathrm{~kg}^{-1} \mathrm{~s}^{-2}\right]$

$H \quad$ Depth of the largest crater identified on the target [m]

$m, M \quad$ Mass of the impactor and that of the target, respectively [kg]

$\Delta M, \Delta M_{c}$ Mass excavated from the crater and from the critical crater that corresponds to catastrophic break up of the target $[\mathrm{kg}]$

$m_{l}, r_{l} \quad$ Mass $[\mathrm{kg}]$ and radius $[\mathrm{km}]$ of the most massive fragment of the target that survived an impact

$Q \quad$ Specific energy provided to the target by the impactor $\left[\mathrm{J} \mathrm{kg}^{-1}\right]$

$Q^{*} \quad$ Specific energy of the catastrophic break up of the target $\left[\mathrm{J} \mathrm{kg}^{-1}\right]$

$R \quad$ Radius of the target [m]

$r, r_{c} \quad$ Radius of the impactor and of the critical-size impactor, respectively [m]

$V \quad$ Volume of the target $\left[\mathrm{m}^{3}\right]$

$\Delta V, \Delta V_{c} \quad$ Volume of the crater, and of the critical crater respectively that corresponds to catastrophic break up of the target $\left[\mathrm{m}^{3}\right]$

$Y \quad$ Impact strength of the target $[\mathrm{Pa}]$

$\delta \quad$ Mean density of the impactor $\left[\mathrm{kg} \mathrm{m}^{-3}\right]$

$\rho \quad$ Mean density of the target $\left[\mathrm{kg} \mathrm{m}^{-3}\right]$

$\psi \quad$ Porosity of the target, dimensionless 


\section{Introduction}

Observations of the solid (icy or rocky) and atmosphere-less bodies of the Solar System indicate that the surfaces of most or even all of them have evolved due to impacts. Thus study of the craters covering the surfaces as well as the morphology of individual craters is crucial for a better understanding of the processes of the evolution of these bodies, particularly small bodies which given their size may be more at risk from impact disruption than are larger bodies. The basic tool to study the impact history of small bodies is to map their surfaces looking for evidence of impact events. So far, various interplanetary spacecraft, mostly different missions to Mars, the Galileo mission to the Jovian system, and the Voyager 1 and 2 missions to the giant planets, have mapped the surfaces of several moons. Hence the topography of the Martian satellites Phobos and Deimos are known rather well. The same holds with the surfaces of the Galilean satellites of Jupiter. The knowledge of smaller Jovian satellites is much less. About $50 \%$ of the surface of each of the medium size satellites of Saturn, Uranus and Neptune are mapped sufficiently detailed (with the resolution of a few kilometres or better) to study the largest craters. In the last decade the amount of data concerning craters on the smaller bodies, especially asteroids, has increased considerably. The NEAR and Hayabusa missions were primarily intended to study asteroids (Eros and Itokawa, respectively). Vesta, the asteroid third in size, was radar-mapped from an Earth based observatory (Mitchell et al., 1996). Other missions have provided targets of opportunities during their cruise phases, e.g. the Galileo mission provided data concerning the asteroid Gaspra as well as Ida and its satellite Dactyl. The current Cassini/Huygens mission is in the process of providing a huge set of data concerning Saturnian satellites, but until this is fully analysed only a few of the smaller icy moons have well recognised craters. In addition, future decades will be needed for new close-up data of Uranian and Neptunian satellites. 
Comprehensive discussion of the limit of catastrophic disruption of solar system bodies was done by Holsapple (1994). His considerations are based on observational data concerning impact cratering available at that time: i.e. Croft (1990) listed the 30 giant impact structures on planets and satellites. The largest target body on this list was Mars, followed by Ganymede, Callisto and the Moon. The smallest bodies were Miranda, Protheus, and Mimas (with radii close to $200 \mathrm{~km}$ ), and finally Phobos, the only body in the Croft's table with a mean radius less than $200 \mathrm{~km}$. An analysis of the largest craters on small bodies was also given by Thomas (1999).

Given the data now available on smaller bodies than those considered by Holsapple (1994) we consider here the influence of relatively large impacts on the evolution of small bodies (diameters of less than a few hundred $\mathrm{km}$ ). We begin by considering the largest craters observed on such objects. We look at both rocky and icy bodies (asteroids and satellites). In the case of the smaller target bodies, the bodies may well be porous or pre-shattered (due to previous impacts). To illustrate this possibility, when discussing observational data for real bodies, the evidence for porosity is given. Although 4 comet nuclei are now observed with good resolution (Halley, Borrelly, Wild-2 and Tempel 1), an impact origin of all the crater-like structures on these bodies is doubtful, although some authors have analysed them as such (e.g. Burchell and Johnson, 2005, Basilevsky and Keller 2007). They could be as well the results of the outgasing processes and are not necessarily post impact structures. Hence, large crater-like structures on cometary nuclei are not considered here.

Once the limit of the largest crater has been passed, shattering of the target body occurs, and, provided sufficient energy is given to the resulting fragments, they can disperse against their own self-gravity. Catastrophic disruption of the targets corresponds to the uppermost limit of the impact studies of cratering. Experimental studies of impact cratering have developed intensively in recent decades, but mostly for rocky target bodies. Accordingly, we present here 
new data for high speed impact disruption of icy targets in the laboratory and compare the results to both models and observational data. Modelling is required as extrapolation of laboratory results concerning decimetre-scale targets to the Solar-System-scale targets is difficult, although several schemes have been proposed. Holsapple 1994 proposed an analytic model, and Benz and Asphaug, 1999 give the results of hydrocode simulations for catastrophic disruption (and references therein); these results expressed as the critical energy density for disruption $Q^{*}$ vs. target radius $R$ are shown on Fig. 1). Successful extrapolation requires: (i) an appropriate scaling law, (ii) an introduction into the model of the gravity of the target body, and (iii) reasonable estimations concerning a set of material parameters. In general, break up of small targets (e.g. laboratory scale targets) depends on the strength of the target material, so it is strength-dominated. Disruption (and dispersion) of larger targets depends on their own gravity, therefore it is gravity-dominated. The small solar system bodies considered here are either in the strength dominated regime or in the transition size range between the two regimes. In the laboratory experiments we ignore porosity and the possible rubble pile nature of small bodies, and address what are in effect pristine, homogeneous, rigid bodies. We compare the outcomes of the models with the laboratory data and comment on the outcome.

\section{The largest craters on small satellites and on asteroids}

In this section we take into account the observational data concerning large craters on small bodies. For the purpose of this work it was possible to list 21 small rocky or icy/rocky bodies, see Table 1 and Fig. 2. Only 4 bodies given in Table 1 have been listed previously by Croft (1990). However, 13 objects (Vesta, Proteus, Mimas, Hyperion, Janus, Amalthea, Epimetheus, Thebe, Mathilde, Ida, Phobos, Deimos, and Gaspra) were considered by Thomas 
(1999). The largest small body considered in Table 1 is the asteroid Vesta with $R=265 \mathrm{~km}$. The smallest one is Dactyl, the satellite of an asteroid Ida, with $R=0.7 \mathrm{~km}$. For our purpose the size (the mean radius $R$ ) of the target body, as well as the diameter $D$ of the largest crater observed on its surface are essential. The target mass $M$, density $\rho$ and porosity $\psi$ are of secondary importance. However, they would be essential if the number of target bodies listed in Table 1 would be considerably greater. In such a case grouping of targets according to their different parameters would be feasible. However, given the small number of objects available here we group them as either icy bodies or rocky bodies. The parameters $R$ and $D$ for all bodies in Table 1 are rather well known. However, their masses $M$ and therefore their mean densities $\rho$ are known with much less accuracy. In some cases they are not known at all. Another problem that emerges concerns the origin of the observed craters: Are all of them impact-originated? A reasonable quick answer is 'yes' for most of the craters on the satellites and on the asteroids. However, the latter can be collisional shards and the depressions observed on their surfaces could be the result of a break-out process of a pristine larger body but not the result of an impact on a given location on a body existing in present. In the case of Arden Coronae on Miranda the association with an impact origin has been questioned as some aspects of the feature require a higher impact speed than expected (McKinnon et al., 1991).

The data in Table 1 allow us to find linear dependences between the diameters $D$ of the largest craters and the target radii $R$. In the formulae below $D$ and $R$ are in kilometres, $q$ is the correlation coefficient, and $n$ is the number of the bodies used for the respective formula. All fits are shown on Fig 2a. For 6 icy satellites (Amalthea, Mimas, Hyperion, Phoebe, Janus, and Epimetheus) there is:

$$
D=6.72+0.813 R, q=0.813, n=6,58.3 \mathrm{~km}<R<198.6 \mathrm{~km} .
$$


For 9 rocky bodies, namely 2 satellites (Phobos, Deimos) and 7 asteroids (Eros, Ida, Mathilde, Gaspra, Juno, Annefrank and Dactyl):

$$
D=1.19+0.838 R, \quad q=0.991, \quad n=9, \quad 0.7 \mathrm{~km}<R<120 \mathrm{~km}
$$

The linear regression formula $D(R)$ for 19 of 21 bodies listed in Table 1 is:

$$
D=-1.90+0.904 R, \quad q=0.957, \quad n=19, \quad 0.7 \mathrm{~km}<R<210 \mathrm{~km}
$$

In formulae $(2)-(3)$ the $1 \sigma$ uncertainty on the intercept is of similar magnitude to the given value of the intercept itself, whereas the uncertainty on the slope is typically only $5-6 \%$ of the slope. In formula (3) Miranda is not taken into account since an impact origin of the geological structure Arden Coronae on Miranda is not certain. Also in formula (3) we do not include Vesta; inclusion in the fit significantly worsens the quality of the fit such that it is no longer an adequate description of the data. It is not clear if this is a somewhat extreme fluctuation or if it is related to the observation that Vesta is considerably more massive and denser than the other bodies listed in Table 1. However, the satellites with doubtful composition (i.e. icy or rocky?) namely Puck, Proteus, Helene and Thebe are taken into account. The high value of the correlation coefficient $q$ in equations (1)-(3) indicates that linear fitting is appropriate. The free terms are insignificant in comparison with terms depending on $R$. So, the diameters of the largest craters on both the icy and rocky bodies fulfil the proportionality

$$
D=(0.90 \pm 0.05) R \text { for } R<210 \mathrm{~km}
$$


We use a similar, linear, approximation for the relation between the diameter of the crater with critical size $D_{c}$ (i.e. on the limit of catastrophic disruption of the target) and $R$. However, the coefficient of proportionality is expected to be larger than those in equation (4). We suggest that the value of this coefficient is nearing to the largest observed value of $D / R$. For the icy Hyperion there is $D / R=1.2$ and for the rocky Deimos $D / R=1.6$. So, the estimates of critical diameters of impact craters are as follow

$$
D_{c, i c y} \approx 1.2 R, \quad D_{c, r o c k y} \approx 1.6 R
$$

It is obvious that an observation of an impact crater on the surface of the target body that was destroyed by that impact is impossible. Therefore, equations (1)-(3) correspond to the large but sub-catastrophic craters. Possibly, the craters on Hyperion and Deimos were produced by the impacts with energies very near to the limit of catastrophic break-up. Thus although the values in (5) are really lower limits they may well be approaching the true case, particularly for the rocky bodies.

Applying the model of crater shape on a finite spherical body shown in the Appendix, we can obtain formulae for the critical volume $\left(\Delta V_{c}\right)$ removed by a critical impact. Formulae (A2 - A4) with $D_{c}$ instead of $D$ and thus $A_{c}$ in place of $A$ permit an estimate of the volume $\Delta V_{c}$ of the critical crater. This formula applied for the icy satellites targets $\left(D_{c}=1.2 R\right.$, therefore $A_{c}$ $=0.8)$ and for the asteroids and rocky satellites targets $\left(D_{c}=1.6 R\right.$, therefore $\left.A_{c}=0.6\right)$ gives respectively

$$
\Delta V_{c, i c y}=0.028 \mathrm{~V}, \quad \Delta V_{c, r o c k y}=0.104 \mathrm{~V}
$$


In the Section 5 the above empirical results will be applied for a discussion of the energy threshold for catastrophic disruption. The material excavated from the crater is typically crushed into many small and even very small pieces. If a catastrophic impact occurs the rest of the target is disrupted into initially just a few large and then into many, numerous small fragments. In the 'limit by definition' of catastrophic impact (in the absence of self gravity which would cause re-assembly) there are only two fragments: the first with mass $m_{l}=M / 2$ and the second with mass $(M / 2-\Delta M) . \Delta M$ is not defined as yet but includes ejected material. Of course, in reality, once the threshold for shattering is exceeded the number of large fragments is greater than two and their masses are smaller, with numerous small fragments also present. The result of an impact can be observed in the laboratory (where the whole spectrum of fragments can be studied - see e.g. Arakawa et al., 2002 for icy bodies) as well as in the Solar System. In the latter case the impact-originated families of asteroids are observed with a size spectrum that is cut off at a certain observational limit.

\section{Laboratory Experiments for Icy Bodies}

Before developing a model for calculation of $Q^{*}$ as a function of $R$, we consider laboratory data for $Q^{*}$ in high speed impacts. Laboratory testing can determine $Q^{*}$ for centimetre to decimetre sized bodies. Accordingly, data are presented here for impacts on icy bodies using a two stage light gas guns (Burchell et al., 1999). Targets were solid (non-porous) ice spheres of negligible porosity and temperature $255 \mathrm{~K}$. Target strength (at low strain rate, $10^{-}$

${ }^{3}-10^{-4} \mathrm{~s}^{-1}$ ) was found by uniaxial compression of ice cylinders (made in similar fashion) along their major axis to be $1.72 \pm 0.93 \mathrm{MPa}$. Using the Brazilian compression method on ice cylinders (where cylinders are compressed transversely across a diameter) the ice tensile strength is estimated as $0.62 \pm 0.18 \mathrm{MPa}$, in both cases comparable to but slightly lower than 
the values of Hiraoka et al., 2006. The targets were made in spheres using thin walled rubber balloons sitting in hemispherical moulds. The resulting spheres were not quite clear, indicating some internal bubbles and possible stresses. However, the strengths obtained from cylinders of ice made in the same fashion were similar to those from cylinders of water ice produced by the procedure described in Grey et al., 2002 and Grey and Burchell 2003.

Three impact programmes were carried out with a total of 27 shots. Shot details are given in Table 2. In programmes $\mathrm{A}$ and $\mathrm{B}$ the ice targets had a diameter of $10-11 \mathrm{~cm}$. In $\mathrm{A}$ the projectiles were $1.5 \mathrm{~mm}$ diameter aluminium spheres, and in B were $0.8 \mathrm{~mm}$ diameter stainless steel spheres. This was to test if projectile density influenced catastrophic disruption. In programme $\mathrm{C}, 0.8 \mathrm{~mm}$ diameter stainless steel projectiles were used again, but with larger targets $(20 \mathrm{~cm}$ diameter) to see if there was a significant effect with changing the ratio projectile: target mass by a factor of $6-8$. In all 3 programmes, impact speed was in the range $1-8 \mathrm{~km} \mathrm{~s}^{-1}$, and by varying the speed the impact energy was varied such that it scanned across a range of values of $Q$.

The mass of the spheres was measured before and after impact. When cratering occurred, the remaining mass of the sphere and the diameter of the crater were measured. If disruption occurred, the mass of the largest fragment was measured. Defining the ratio $m_{l} / M$ as the remaining mass of the sphere (or mass of the largest fragment after impact) divided by the pre-impact target mass $M$, the variation of $m_{l} / M$ with $Q$ is shown in Fig. 3. In general, at low $Q$ values, cratering occurs and $m_{l} / M$ is close to one. At a critical value $Q^{*}$, a crater forms and disruption occurs, marked by a sudden fall in the mass ratio. Thus part of the target is lost as crater ejecta, and the remaining mass falls into two near equal sized pieces, each just under 0.5 times the original mass. At higher $Q$ values the mass ratio continues to fall as the largest surviving fragment decreases in size. For $Q$ much less than $Q^{*}$, we observe that craters have the typical appearance of impact craters in ices (e.g. Grey et al., 2002). These are pit shaped 
central craters surrounded by a much wider shallow (spallation) zone. As $Q$ approaches $Q^{*}$ we observe that the craters appear much flatter, as if a slice had been removed from the front of the spherical targets. In cases where we just exceed $Q^{*}$, we have obtained two near equal sized fragments which can be reconstructed into a single object which resembles the targets from impacts where $Q$ is just under $Q^{*}$ but with slighter larger diameter craters. Any removal of material from the rear of the target is negligible compared with that arising from the crater at the impact point. The disruption of the target when $Q=Q^{*}$ is thus occurring by fracturing of the body, and not by bulk excavation of material.

In Programme A, the data all have $Q \geq Q^{*}$. A fit of a power law to the data of the form:

$$
m_{l} / M=a Q^{b}
$$

yielded values for $a$ and $b$, along with $q$ (the correlation coefficient) as given in Table 3. If this fit is extrapolated to $m_{l} / M=0.5$, this gives a $Q^{*}$ of $(1.9 \pm 0.3) \mathrm{J} \mathrm{kg}^{-1}$.

In Programme B, the data span both regimes, cratering and disruption. The two regimes are in general very different in nature, but some scatter in the data means there is an overlap region between the two regimes. If the mean of this overlap is taken as $Q^{*}$, with the extremes giving the range of uncertainty, we obtain $Q^{*}=(1.74 \pm 0.18) \mathrm{J} \mathrm{kg}^{-1}$. This is compatible with the value found in Programme A with different projectiles. We also fit the data for $m_{l} / M$ in the fragmentation regime and obtain values for $a$ and $b$ as given in Table 3. If we use the fit results to predict $Q^{*}$ (as for Programme A) we obtain $Q^{*}=(1.2 \pm 0.4) \mathrm{J} \mathrm{kg}^{-1}$, compatible with the value by directly finding the transition region on the graph. For the data in the cratering regime in Programme B, we measured the diameter of the observed craters. These are given in Table 4, along with the ratio of crater diameter $D$ to target radius $R$. This ratio $D / R$ would be 2 if a crater 
extended across the whole of a target. The values for $D / R$ are plotted in Fig. 4 (as open circles). It can be seen that as $Q$ approaches the value of $Q^{*}$, the value of $D / R$ rises sharply to 1.64 .

In Programme C, the data (Fig. 3c) almost all lie in the cratering regime. There is then a sudden jump to disruption and fragmentation. Taking $Q^{*}$ as lying between the last two data points (where $m_{l} / M$ falls suddenly to less than 0.5$)$, we obtain $Q^{*}=(18.0 \pm 0.7) \mathrm{J} \mathrm{kg}^{-1}$. This is an order of magnitude greater than in the other two experimental programmes. No experimental reason was found for this, although we note that a higher impact speed was used in this programme. We again measured the crater diameters (Table 4) and these are also plotted in Fig. 4 (as filled squares). For a wide range of $Q$ values, $D / R$ seems stable at just over 1 , but as $Q$ approaches $Q^{*}$ the ratio rises suddenly to a maximum of 1.5 .

Previously, Arakawa (1999) fired nylon projectiles at similar speeds (2.3 to $\left.4.7 \mathrm{~km} \mathrm{~s}^{-1}\right)$ onto ice targets and obtained $Q^{*} \approx 34 \mathrm{~J} \mathrm{~kg}^{-1}$, with $a=(13 \pm 6)$ and $b=-(0.91 \pm 0.08)$. The value of $b$ is compatible with those found here, although the absolute magnitude of $Q^{*}$ is noticeably greater than that in Programmes A and B. However, given an uncertainty of almost $50 \%$ in the value of $a$, this is compatible with the results of programme C. In separate work with low speed impacts on ice (150 to $670 \mathrm{~m} \mathrm{~s}^{-1}$, nylon projectiles), Arakawa et al., 2002 found $Q^{*}=20.7 \mathrm{~J} \mathrm{~kg}^{-1}$. At even lower speeds $\left(108\right.$ to $\left.182 \mathrm{~m} \mathrm{~s}^{-1}\right)$ impacts of aluminium projectiles on ice spheres were reported by Ryan et al., 1999. From 3 events they found $Q^{*} \approx 10 \mathrm{~J} \mathrm{~kg}^{-1}$ and by fitting their data we obtain $a=(8.5 \pm 9.5)$ and $b=-(1.2 \pm 0.4)$ with $q=0.83$. However, in a later paper by the same authors (Giblin et al., 2004), it is reported that in the original paper an artefact of the target production mechanism may have weakened the targets resulting in an artificially low measure of $Q^{*}$.

Overall there is some scatter on the values with $Q^{*}$ for icy bodies, and it is not clear if this is due to different impact conditions or some intrinsic scatter due to varying target 
production etc. Nevertheless, the range of $Q^{*}$ observed for impacts on solid ice targets is constrained to lie between 1 and a few tens of $\mathrm{J} \mathrm{kg}^{-1}$.

The values of $Q^{*}$ obtained here, and in previous work as quoted, are all significantly lower than those obtained by modelling such as that of Benz and Asphaug (1999). In their work, Benz and Asphaug considered impacts on ice spheres, and at $3 \mathrm{~km} \mathrm{~s}^{-1}$, for targets $10 \mathrm{~cm}$ across predicted $Q^{*}$ of some $400 \mathrm{~J} \mathrm{~kg}^{-1}$ (see Fig. 5 for a comparison of laboratory results for $\mathrm{Q}^{*}$ and the model predictions). This is significantly greater than the values found in any experimental laboratory work at these size scales, indicating the need for further modelling. They note however that more work still needs to be done to obtain better material properties for icy bodies at high strain rate. This may lead to inaccuracies in modelling in the strength dominated regime. In addition, we note that in Benz and Asphaug the behaviour of the ratio $m_{l}$ $/ M$ can be fit as per eqn. 7 here, but with a single value of the power over the whole range of $Q$ (see for example fig. 9b in Benz and Asphaug). At laboratory scales this is generally not the case for ice, where a change in slope (i.e. a change in the value of the power in eqn. 7 here) is usually observed.

As well as determining $Q^{*}$, the data permit a consideration of the ratio crater diameter to target radius $(D / R)$ for the cratering (as distinct from catastrophic disruption) events. The $D / R$ ratio for the three cratering events in Fig. 4 was found to be $1.19,1.36$ and 1.64. Given that the magnitude of $D / R$ is suddenly increasing as $Q$ approaches $Q^{*}$, it is not clear if $D / R$ can exceed 1.64 or if this is the true limit, with crack propagation in the target weakening it and causing disruption (see above). In the case of disrupted targets with $Q$ around $Q^{*}$, when (as stated) we reassembled the target from the 2 largest fragments, the observed crater on the front had $D / R$ slightly less than 2 .

Thus overall, we obtain experimentally that $D_{c}=1.64 R$ for icy bodies. Given that the scales are significantly different between laboratory and Solar System bodies it is not 
immediately apparent how the laboratory result can be scaled to Solar System objects. Further, the Solar System bodies may not be pure water ice and are at various temperatures which are not similar to those used here (see Grey and Burchell 2003 for a discussion of how, at laboratory scales, cratering in ices changes as a function of ice temperature). Accordingly all we can do is note that the limit found from observations (formula $(5)$ ) is $D_{c, i c y} \approx 1.2 R$. Given that the set of observations cannot be considered complete, with more small icy bodies still to be fully mapped, the current observational result should be considered a lower limit.

\section{Large craters on small bodies as a sub-threshold of catastrophic break up}

Here, since the target bodies are small, craters are formed in the strength dominated regime. Since the transition between simple and complex crater morphologies is gravity (and hence target body size) dependent (e.g. see Holsapple 1994), even the largest possible craters on small bodies (a few hundred $\mathrm{km}$ or less) will be simple craters. Most of the impact craters observed on the bodies of the Solar System are axially symmetric with reasonably well formed borders. Let $D_{c}$ be the diameter of the largest crater that can be formed on a target body without shattering that body and hence still be observed at a later date. In other words, $D_{c}$ corresponds to the most energetic impact that can be sustained by a target with radius $R$ without its break up. For small bodies (neglecting self gravity) a marginally more energetic impact would indeed catastrophically break up and disperse the body, but for (larger) gravitationally bound bodies reassembly would re-occur after such an impact until sufficient additional energy was supplied in still higher energy impacts to disperse the fragments against gravity.

Let $\Delta V$ and $\Delta V_{c}$ be the volumes excavated from the crater with diameter $D$ and $D_{c}$, respectively. Since $\Delta V_{\mathrm{c}}$ is the upper limit of the crater volume on a given target therefore the energy for formation of the crater $D_{c}$ is equal to the lower limit of target break up energy $E_{c}$. Of 
course, by definition of $D_{c}$, for any observed crater with diameter $D$ there is $D \leq D_{c}$ and $\Delta V<$ $\Delta V_{c}$.

On the basis of data collected in Table 1 we deduce some regularities concerning large craters on the small bodies in Solar System. Unlike for a crater on a flat surface (Fig. 6a) a cross section of a crater by a plane perpendicular to the target surface and containing the crater axis can have different convexity, as is schematically presented on Fig. 6b-d. In the following we assume that all the craters have a flat bottom, e.g. Fig. 6d (and as was indicted in the laboratory results for the largest craters on spherical targets). So, the geometry of the craters is fully defined by only one parameter, namely the ratio $D / R$. (see Appendix, formulae A1 - A2). This is obviously a crude simplification. However, it can be justified on the basis of the experiments performed in laboratory which show similar behaviour. As it is shown in the Table 1 the characteristic values of the ratio $D / R$ for the large craters on the small bodies of the Solar System belong to an interval $0.9-1.6$. To that interval corresponds the ratio $H / D$ (where $H$ is crater depth) from an interval $0.12-0.25$. The laboratory data of Burchell et al., 2005, for bowl-shaped craters formed on a flat icy surface have $H / D$ values of $0.12-0.27$ for $\mathrm{H}_{2} \mathrm{O}$ ice targets, and $0.09-0.31$ for $\mathrm{CO}_{2}$ ice targets. For rocky targets the ratio $H / D$ is $0.18-0.20$ (Gault 1973) or similarly 0.16-0.26 (Burchell and Whitehorn, 2003).

The model of the flat-bottom crater on the sphere allows for simple comparison of different craters on different bodies. For a flat-bottom crater, the material of an impacted target initially formed (before it was thrown-out) a segment of a sphere. Then the impact cut-off the segment and threw the material away. The assumption that material excavated from the crater is also removed from the target is not strictly true at scales where gravity effects cannot be neglected. Polansky and Ahrens (1990) reported that at laboratory scales, of order $50 \%$ of impact ejecta in high speed impacts had a maximum ejection speed of $30 \mathrm{~m} \mathrm{~s}^{-1}$. Similarly, Hartmann (1985) reported that 50\% of ejecta had speeds above $64 \mathrm{~m} \mathrm{~s}^{-1}$. These values are for 
impacts into solid targets. At higher energies and larger crater radii, the absolute ejecta velocity values will increase (see Hartmann 1985, or Holsapple 1993 for a discussion of general scaling laws for ejecta). For bodies of radius order $100 \mathrm{~km}$, we can naively state that $\mathrm{v}_{\mathrm{esc}}\left(\mathrm{m} \mathrm{s}^{-1}\right) \sim R$ $(\mathrm{km})$. Thus of order $50 \%$ of the crater mass will be ejected at close to or above escape velocity even for targets of size $100 \mathrm{~km}$ and the approximation is not unreasonable. The remaining material will be distributed across the target body as a regolith like blanket. In addition, we note that if the whole target consists of non-cohesive small scale material, then the efficiency of ejection will fall due to lower ejection velocities (e.g. Hartmann 1985, Wada et al., 2005). This cutting-off forms a flat-bottom crater on a spherical globe. It corresponds to the bowl-shaped crater formed on a flat surface. The volume of the segment of the sphere and therefore the volume of the crater $\Delta V$ as well as the mass excavated from the crater $\Delta M$ (Fig. 6d) are derived in Appendix A (formulae A3 - A4) and if the body then reassembles under gravitational influence as a sphere the reduced radius and mass are given by formulae A5 - A6.

Formulae A1 - A6 allow us to treat the largest craters on the different targets as geometrically similar. The volume of the crater produced by a vertical impact on a uniform spherical target depends on (at least) five dimensional parameters (e.g. Melosh 1989, Housen and Holsapple 2003). They are the impactor energy $E$, two of the three target parameters $(\rho, M$, $R$ ), the strength $Y$ of the target material and the gravity constant $G$.

We imagine that: (i) In reality the observed crater is not the largest one that can be formed on the target body, and (ii) The spherical-segment crater form is a crude approximation. The former assumption can only be overcome with more complete mapping of more icy bodies. As has already been noted, this may result in an increase in the values in equation 5 which will probably be only a modest increase as some bodies have already been mapped. To overcome the approximation expressed by the point (ii) one should really consider the largest crater separately for each of the considered bodies. To achieve this, detailed 3 dimensional 
maps would be needed of all such craters observed, which is not plausible for most craters. So, our approximations seem to be reasonable.

\section{Conclusions}

In general, models based on basic physics and material mechanics allow predictions of $Q^{*}$ as a function of target body radius. The sensitivity of the predictions to variation of the impact conditions and parameters in the models (e.g. impact speed, angle of incidence, material strength etc.) show that excavation energy and fragment size distribution influence $Q^{*}$ for target body radius up to $100-1000 \mathrm{~m}$ (e.g. see Fig. 1). Above this size the self-gravitational term dominates, impeding dispersion of the fragments after impact. The exact size at which the transition from one regime to the other occurs depends on the models. Some of the small icy bodies considered in this paper lie in the general size regime where this transition occurs in the icy body model of Benz and Asphaug (1999). Indeed, although not considered here, some of the medium sized icy satellites also fall into this size range (e.g. see Moore et al., 2004 for a discussion of cratering on medium sized icy satellites).

Laboratory data for impact disruption of icy bodies shows that the largest crater possible forms on the target which then disrupts. The diameter of this largest possible crater is some $80 \%$ of the radius of the target. This compares to a value of about $60-70 \%$ for the largest observed craters on small Solar System icy bodies. In addition the laboratory results for $Q^{*}$ are compared with the hydrocode results of Benz and Asphaug (1999). A significant difference in magnitude of $Q^{*}$ is found at decimeter scales between the laboratory and model results. It is not clear if this is just an overall overestimate of $Q^{*}$ in the model (which could therefore just be scaled to match the laboratory results), or if there is a more subtle effect that needs correction. If the latter is true, then the conclusion of Benz and Asphaug that the weakest 
ice bodies in the Solar system are of radius $100-200 \mathrm{~m}$ (for impact speeds between 0.5 and 3 $\mathrm{km} \mathrm{s}^{-1}$ ) may need to be revisited.

It remains to extend this work to the case of non-zero porosity. This will be increasingly important as smaller and smaller bodies (more prone to impact disruption) are considered. Such bodies will generate smaller internal pressures under their self gravitation and can thus have a non-negligible porosity. As indicted here (Table 1), there is evidence that porosity is already showing up at the size scales considered here. Two types of porosity will have to be considered, which have different influences on the outcome of collisions and transmission of shock pressure waves: Micro porosity (i.e. where pore space is small on the scale of the body involved and the pores are distributed throughout the body) and macro-porosity (where large voids are distributed throughout the body which is otherwise made of relatively non-porous materials). For micro-porosity, at present both hydrocode modeling (e.g. Wünnemann et al., 2006, Wada et al., 2006) and laboratory impact data (e.g. Arakawa et al., 2002, Housen and Holsapple 2003) for impacts all show the importance of energy flowing into pore space compression when considering impact events. The introduction of micro-porosity into target bodies thus reduces the size of the resultant craters, and may correspondingly act to increase the required $Q^{*}$ (see Burchell et al., 2005 for examples of growth of $Q^{*}$ with increasing porosity in laboratory experiments). Macro-porosity (e.g. a rubble pile nature for a body) on the other hand influences the propagation of shock waves through the bulk of a body and may thus play a significant role in determining $Q^{*}$ which can be driven by large scale fracturing processes throughout a body. As a result, both effects of this should be incorporated in future modeling work which aims to provide a full picture of $Q^{*}$ for small to medium sized solar system bodies. At present ad-hoc adjustments to the results are possible. For example, when considering laboratory scale data, various authors such as Love et al., 1993 and Burchell et al., 2005, propose scaling of the critical energy density as a function of porosity. However, caution 
is needed as the exact nature of such scaling for target sizes above those in the laboratory remains to be determined and whilst one type of porosity (here micro-porosity) may be allowed for, the second type (macro porosity still needs to be considered).

Acknowledgments. This work was partially supported by grant no. 0576/H03/2007/32 provided by the Polish Ministry of Education and Science. MJB thanks the Science and Technology Facilities Council (UK) for support and A. Lightwing for measuring the ice strengths. We thank the Reviewers for their constructive criticism and useful comments.

\section{References}

Arakawa, M. 1999. Collisional Disruption of Ice by High-Velocity Impact. Icarus 142, 34-45.

Arakawa, M., Leliwa-Kopystyński, J., Maeno, N. 2002. Impact experiments on porous icysilicate cylindrical blocks and the implication for disruption and accumulation of small icy bodies. Icarus 158, 516-531.

Baliunas, S., Donahue, R., Rampino, M. R., Gaffey, M. J., Shelton, J., C., Mohanty, S. 2003. Multispectral analysis of Asteroid 3 Juno taken with the 100-inch telescope at Mount Wilson Observatory. Icarus 163, 135-141.

Basilevsky, A. T., Keller, H. U. 2007. Craters, smooth terrains, flow and layering on the comet nuclei. Solar System Research 41, 109 - 117.

Benz, W., Asphaug, E. 1999. Catastrophic disruption revisited. Icarus 142, 5-20.

Britt, D. T., Yeomans, D., Housen, K., Consolmagno, G. 2002. Asteroid density, porosity, and structure. In Bottke, W. F., Cellino, A., Paolicchi, P., Binzel, R. (Eds.), Asteroids III, Univ. Arizona Press, Tucson, pp. 485-500. 
Burchell, M. J., Cole M. J., McDonnell J. A. M., Zarnecki J. C. 1999. Hypervelocity impact studies using the $2 \mathrm{MV}$ Van de Graaff accelerator and two-stage light gas gun of the University of Kent. Meas. Sci. Technol., 40, 41-50.

Burchell M.J., Whitehorn L. 2003. Oblique incidence hypervelocity impacts on rock. Monthly Notices of the Royal Astronomical Society 341, 192-198.

Burchell M.J., Johnson E. 2005. Impact Craters on Small Icy Bodies, i.e. Icy Satellites and Comet Nucleus. Monthly Notices of the Royal Astronomical Society 360, 769-781.

Burchell, M. J., Leliwa-Kopystyński, J., Arakawa, M. 2005. Cratering of icy targets by different impactors: laboratory experiments and implications for cratering in the solar system. Icarus $179,274-288$.

Croft, S. K. 1990. Observational limits on catastrophic disruption. Lunar Planetary Sci. XXIII, 269-270.

Duxbury, T.C., Newburn, R. L., Acton, C. H., Carranza, E, McElrath, T. P., Ryan, R.E., Synnott, S. P., You, T. H., Brownlee, D. E., Cheuvront. A. R., Adams, W. R., ToroAllen, S. L., Freund, S., Gilliland, K. V., Irish, K. J., Love, C. R., McAllister, J. G., Mumaw, S. J., Oliver, T. H., Perkins, D. E. 2004. Asteroid 5535 Annefrank size, shape, and orientation: Stardust first results. J. Geophys. Res., Vol. 109, No. E2, E02002.

Gault D.E. 1973. Displaced mass, depth, diameter and effects of oblique trajectories for impact craters formed in dense crystalline rocks. The Moon 6, $32-44$.

Giblin, I., Davis, D. R., Ryan, E. V. 2004. Collisional disruption of porous icy targets simulating Kuiper belt objects. Icarus 171, $487-505$. .

Grey, I. D. S., Burchell, M. J. 2003. Hypervelocity impact cratering on water ice targets at temperatures ranging from $100 \mathrm{~K}$ to $253 \mathrm{~K}$. Journal of Geophysical Research 108(E3), 5019. 
Grey, I. D. S., Burchell, M. J., Shrine N. R. G. 2002. Scaling of hypervelocity impact craters in ice with impact angle. Journal of Geophysical Research 107(E10), 5076.

Hartmann, W. K. 1985. Impact experiments.1 Ejecta velocity distributions and related results from regolith targets Icarus 63, $69-98$.

Hiraoka, K., Arakawa, M., Seto, M., and Nakamura A. M. 2006. Measurement of compressive and tensile strength of ice-silicate mixtures. Lunar and Planetary Science Conference XXXVII, abstract 1601.

Holsapple, K. A. 1993. The Scaling of Impact Processes. Annual reviews of Earth and Planetary Science 21, 333-373.

Holsapple, K. A. 1994. Catastrophic disruption and cratering of solar system bodies: a review and new results. Planet. Space Sci., Vol. 42, No.12, 1067-1078.

Housen K. R., and Holsapple K. A. 2003. Impact cratering on porous asteroids. Icarus 163, 102 $-119$.

Love S.G., Hörz F., Brownlee D.E. 1993. Target porosity effects in impact cratering and collisional disruption. Icarus 105, $216-224$.

McKinnon, W.B., Chapman, C.R., Housen, C.R. 1991. Cratering of the Uranian Satellites. In: Bergstrahl, J.T., Miner, L.D., Matthews, M.S. (Eds.), Uranus. Univ. of Arizona Press, Tucson, pp. 2967-2982.

Melosh, H. J. 1989. Impact cratering. Series: Oxford Monograph on Geology and Geophysics, \#11. Oxford University Press.

Mitchell D. L., Ostro S. J., Hudson R. S., Rosema K. D., Cambell D.B., Vélez, Chandler J. F., Shapiro I. I., Giorgini J. D., and Yeomans D. K. 1996. Radar Observations of Asteroids 1 Ceres, 2 Pallas, and 4 Vesta. Icarus 124, 113-133. 
Moore, J. M., Schenk, P. M., Bruesch, L. S., Asphaug, E., McKinnon, W. B. 2004. Large impact features on middle-sized icy satellites. Icarus 171, 421-443.

Polansky, C. A., Ahrens, T. J. 1990. Impact spallation experiments: fracture patterns and spall velocities. Icarus $87,140-155$.

Ryan, E. V., Davis D. R, and Giblin I. 1999. A laboratory impact study of simulated Edgeworth-Kuiper belt objects. Icarus 142, 56-62.

Thomas, P. C., Binzel, R. P., Gaffey, M. J., Storrs, A. D., Wells, E. N., Zellner, B. H. 1997. Impact Excavation on Asteroid 4Vesta: Hubble Space Telescope Results. Science, Vol. 277 , no. $5331,1492-1495$.

Thomas, P. C. 1999. Large craters on small objects: occurrence, morphology and effects. Icarus $142,89-96$.

Wada, K., Senshu, H., Matsui, T. 2005. Numerical simulation of impact cratering on granular material. Icarus $180,528-545$.

Wünnemann, K., Collins, G.S., Melosh, H.J. 2006. A strain-based porosity model for use in hydrocode simulations of impacts and implications for transient crater growth in porous targets. Icarus $180,514-527$. 


\section{Appendix: Large craters on finite (relatively small) bodies}

Assume a sphere of radius $R$, a crater of diameter $D$ and depth $H$. Assume that the magnitude of $D$ approaches $R$, and that due to sideways growth of the crater in a finite curved body the crater floor becomes flat. The crater is thus effectively a slice removed from the front of the spherical target. Then geometry requires:

$$
\begin{gathered}
H / D=(R / D)(1-A) \\
\text { where } A=\sqrt{1-0.25(D / R)^{2}}
\end{gathered}
$$

Thus the crater shape (i.e. depth / diameter, $H / D)$ is constrained by only one ratio $(D / R)$. Further, the excavated volume $\Delta V$ is given by

$$
\Delta V=\left(0.5-0.75 A+0.25 A^{3}\right) V
$$

And the excavated mass $\Delta M$ by

$$
\Delta M=\rho \Delta V
$$

Accordingly the radius of the surviving body is given by

$$
R-\Delta R=R\left(0.5+0.75 A-0.25 A^{3}\right)^{1 / 3},
$$

and the surviving mass by

$$
\left.M-\Delta M=\rho V\left[0.5+0.75 \mathrm{~A}-0.25 A^{3}\right)\right] .
$$

For the critical case (i.e. a just disrupted target) the subscript $\mathrm{c}$ is applied to the relevant parameters in the above relations (i.e. $H, D, A$ and $r$ are replaced by $H_{c}, D_{c}, A_{c}$ and $r_{c}$ ). 


\section{Figure captions.}

Figure 1. Examples of prediction for specific energy for catastrophic disruption $Q^{*}$ in $\mathrm{J} \mathrm{kg}^{-1}$ versus target radius $R$ in meters. Cases (a)-(d) according to Benz and Asphaug (1999): (a) and (b) basalt target shattered by projectile with velocity $5 \mathrm{~km} \mathrm{~s}^{-1}$ and $3 \mathrm{~km} \mathrm{~s}^{-1}$, respectively; (c) and (d) icy target shattered by projectile with velocity $3 \mathrm{~km} \mathrm{~s}^{-1}$ and $0.5 \mathrm{~km} \mathrm{~s}^{-1}$, respectively. Note that according to Benz and Asphaug (1999) increasing the impact velocity decreases $Q^{*}$ for basalt but it increases $Q^{*}$ for ice. Case (e) according to Holsapple (1994) and is general curve for minor bodies based on modelling and observation for both icy and rocky bodies.

Figure 2. Relationship between largest crater diameter and satellite radius. See Table 1 for details. (a) Crater diameter $D$ vs. object radius $R$ (fit lines are as in text). (b) Ratio of $D / R$ vs. object radius $R$.

Figure 3. Surviving mass fraction vs. impact energy density in (a) Programme A, (b) Programme B, and (c) Programme C.

Figure 4. Crater diameter normalized to target radius $D / R$ versus impact energy density $Q$.

Figure 5. Comparison of $Q^{*}$ for icy bodies in the laboratory with the hydrocode model results of Benz and Asphaug (1999) (solid and dashed lines represent different impact speeds).

Figure 6. Schematic views of the crater cross sections: (a) on a flat target; (b)-(d) on a spherical target: (b) Convexity directed inward the target, (c) Convexity directed outward the target, and (d) Flat bottom of the crater. The case (b) corresponds to bowl shaped and not very large craters commonly observed on many satellites as well as on the on the asteroids. The case (c) corresponds to very large impact structures identified e.g. on the Moon, on Ganymede, or 
on Vesta. The case (d) is an intermediate structure considered in this paper. In that case the crater volume is given by equation (A3). See text for details.

Table 1. Craters on satellites and asteroids. See also Fig. 1. Radii and densities of the satellites are from http://ssd.jpl.nasa.gov/?sat_phys_par (July 2006 update). The satellites from Amalthea to Miranda are the icy bodies. Their ice to mineral ratio can be roughly supposed to be one to one by mass. The low density satellites are certainly porous. The composition of the satellites Puck, Proteus, Helene and Thebe is not known, so their densities are assumed. Phobos, Deimos and asteroids are rocky. The radii of the bodies listed in the table are known with good accuracy but the knowledge concerning their density is much less. Vesta data are according to Thomas et al., 1997. Juno data are from Baliunas et al., 2003. Annefrank data are from Duxbury et al. 2004. The craters' diameters are from the above sources, or (for Phoebe, Miranda, Puck, Helene, Eros, and Dactyl) they are estimated on the basis of the photos published by NASA. Mean porosities $\psi$ of the icy satellites are estimated by means of formula (8) with assumed composition $C=0.5$ and densities $\rho_{i}=940 \mathrm{~kg} \mathrm{~m}^{-3}, \rho_{r}=3000 \mathrm{~kg} \mathrm{~m}^{-3}$. Mean macroporosities of the rocky bodies are according to Britt et al., (2002, p. 493) where Phobos, Deimos and Mathilde are classified as rubble-pile bodies.

\begin{tabular}{|c|c|c|c|c|c|c|}
\hline Target & $R, \mathrm{~km}$ & $\rho, \mathrm{kg} \mathrm{m}^{-3}$ & $D, \mathrm{~km}$ & $D / R$ & $\psi$ & Remarks \\
\hline Amalthea (Jupiter) & 83.45 & $849 \pm 199$ & 88 & 1.055 & 0.4 & Crater Pan \\
\hline Mimas (Saturn) & 198.6 & $1152 \pm 27$ & 145 & 0.730 & 0.19 & Crater Herschel \\
\hline Hyperion (Saturn) & 133 & $569 \pm 108$ & 163 & 1.226 & 0.2 & - \\
\hline Phoebe (Saturn) & 107.3 & $1633 \pm 49$ & 100 & 0.932 & 0.0 & - \\
\hline Janus (Saturn) & 88.8 & $612 \pm 62$ & 50 & 0.563 & 0.5 & - \\
\hline Epimetheus (Sat.) & 59.5 & $634 \pm 102$ & 40 & 0.672 & 0.6 & - \\
\hline Miranda (Uranus) & 235.8 & $1201 \pm 137$ & 340 & 1.442 & 0.16 & Arden Coronae \\
\hline Puck (Uranus ) & 81 & 1300 ass. & 45 & 0.555 & - & Icy? \\
\hline Proteus (Neptune) & 210 & 1300 ass. & 210 & 1.000 & - & Icy? \\
\hline Helene (Saturn) & 16.3 & 1500 ass. & 10 & 0.613 & - & Icy? \\
\hline Thebe (Jupiter) & 49.3 & 3000 ass. & 44 & 0.892 & - & Rocky? \\
\hline Phobos (Mars) & 11.1 & $1867 \pm 76$ & 9.4 & 0.847 & $0.41-0.46$ & Crater Stickney \\
\hline Deimos (Mars) & 6.2 & $2247 \pm 251$ & 10 & 1.613 & $0.26-0.74$ & - \\
\hline 4 Vesta & 264.5 & $3440 \pm 120$ & 460 & 1.739 & $<0.01$ & - \\
\hline 433 Eros & 8.68 & $2670 \pm 30$ & 5 & 0.576 & 0.18 & Crater Himeros \\
\hline 243 Ida & 15.7 & $2600 \pm 500$ & 13.8 & 0.879 & $0.17-0.25$ & Crater Lascaux \\
\hline 253 Mathilde & 26.5 & $1300 \pm 200$ & 33.4 & 1.260 & $0.35-0.46$ & Crater Karoo \\
\hline 951 Gaspra & 6.2 & n.a. & 3 & 0.48 & - & Neujmin Regio \\
\hline 3 Juno & $\sim 120$ & n.a. & $\geq 100$ & $\sim 0.83$ & - & - \\
\hline
\end{tabular}




\begin{tabular}{|l|c|c|c|c|c|l|}
\hline 5535 Annefrank & $\sim 3$ & n.a. & $\sim 1.5$ & $\sim 0.50$ & - & - \\
\hline Dactyl (Ida's sat.) & $\sim 0.7$ & n.a. & $\sim 0.6$ & $\sim 0.857$ & - & - \\
\hline
\end{tabular}


Table 2. Details of impact programmes

\begin{tabular}{|c|c|c|c|c|c|}
\hline Programme & $\begin{array}{l}\text { Impact } \\
\text { speed } \\
\left(\mathrm{km} \mathrm{s}^{-1}\right)\end{array}$ & $\begin{array}{l}\text { Target } \\
\text { mass } M \\
(\mathrm{~kg})\end{array}$ & $Q\left(\mathrm{~J} \mathrm{~kg}^{-1}\right)$ & $\begin{array}{c}\text { Mass of target or } \\
\text { largest fragment } \\
\text { after impact } m_{l} \\
(\mathrm{~kg})\end{array}$ & $m_{l} / M$ \\
\hline \multirow{12}{*}{ A } & 0.955 & 0.952 & 2.44 & 0.364 & 0.382 \\
\hline & 0.974 & 0.938 & 2.34 & 0.456 & 0.486 \\
\hline & 0.967 & 1.201 & 2.30 & 0.491 & 0.409 \\
\hline & 1.07 & 1.029 & 2.82 & 0.328 & 0.319 \\
\hline & 1.10 & 0.790 & 2.98 & 0.162 & 0.201 \\
\hline & 1.49 & 0.784 & 5.47 & 0.135 & 0.172 \\
\hline & 1.66 & 1.145 & 5.96 & 0.154 & 0.134 \\
\hline & 1.83 & 1.028 & 8.06 & 0.142 & 0.138 \\
\hline & 1.99 & 0.917 & 10.7 & 0.092 & 0.100 \\
\hline & 2.20 & 0.860 & 13.9 & 0.082 & 0.095 \\
\hline & 2.91 & 0.729 & 20.9 & 0.017 & 0.023 \\
\hline & 3.54 & 0.962 & 30.9 & 0.024 & 0.025 \\
\hline \multirow{8}{*}{ B } & 1.05 & 0.820 & 1.43 & 0.781 & 0.952 \\
\hline & 1.12 & 0.695 & 1.92 & 0.636 & 0.915 \\
\hline & 1.16 & 0.955 & 1.50 & 0.924 & 0.968 \\
\hline & 1.20 & 0.983 & 1.56 & 0.495 & 0.504 \\
\hline & 1.23 & 1.030 & 1.56 & 0.338 & 0.328 \\
\hline & 1.52 & 0.913 & 2.67 & 0.231 & 0.253 \\
\hline & 1.56 & 1.181 & 2.19 & 0.235 & 0.199 \\
\hline & 2.17 & 0.919 & 5.45 & 0.167 & 0.182 \\
\hline \multirow{7}{*}{$\mathrm{C}$} & 1.12 & 3.632 & 0.37 & Not recorded & - \\
\hline & 2.90 & 2.999 & 2.98 & 2.925 & 0.975 \\
\hline & 5.01 & 3.633 & 7.34 & 3.559 & 0.972 \\
\hline & 7.04 & 3.571 & 14.8 & 3.507 & 0.982 \\
\hline & 7.50 & 3.670 & 16.3 & 3.354 & 0.914 \\
\hline & 7.84 & 3.498 & 18.7 & 0.815 & 0.233 \\
\hline & 7.88 & 3.824 & 17.3 & 3.596 & 0.940 \\
\hline
\end{tabular}


Table 3. Results of fits to $m_{l} / M=a Q^{b}\left(Q>Q^{*}\right.$ only).

\begin{tabular}{cccccc}
\hline Programme & $\begin{array}{c}\text { Mass ratio } \\
\text { Impactor/ } \\
\text { Target } \\
m / M\end{array}$ & $Q^{*}\left[\mathrm{~J} \mathrm{~kg}^{-1}\right]$ & $a$ & $b$ & $\begin{array}{c}\text { Correlation } \\
\text { coefficient } q\end{array}$ \\
\hline $\mathrm{A}$ & $4.93 \times 10^{-6}$ & $1.9 \pm 0.25$ & $1.01 \pm 0.18$ & $-1.08 \pm 0.17$ & 0.957 \\
$\mathrm{~B}$ & $2.12 \times 10^{-6}$ & $1.77 \pm 0.25$ & $0.58 \pm 0.19$ & $-0.87 \pm 0.48$ & 0.768 \\
$\mathrm{C}$ & $7.08 \times 10^{-7}$ & $18.15 \pm 0.5$ & ---- & --- & ---- \\
\hline
\end{tabular}


Table 4. Experimental data in programmes B and C.

\begin{tabular}{ccccc}
\hline Programme & $\begin{array}{c}\text { Specific } \\
\text { energy of } \\
\text { impact } Q \\
{\left[\mathrm{~J} \mathrm{~kg}^{-1}\right]}\end{array}$ & $\begin{array}{c}\text { Target radius } \\
R[\mathrm{~m}]\end{array}$ & $\begin{array}{c}\text { Crater diameter } \\
D[\mathrm{~m}]\end{array}$ & $\begin{array}{c}\text { Crater diameter } \\
\text { target radius } \\
D / R\end{array}$ \\
\hline \multirow{3}{*}{$\mathrm{B}$} & 1.43 & 0.055 & 0.075 & 1.36 \\
& 1.92 & 0.055 & 0.09 & 1.64 \\
& 1.50 & 0.059 & 0.07 & 1.18 \\
\hline & 0.37 & 0.100 & 0.070 & 0.70 \\
& 2.98 & 0.095 & 0.104 & 1.10 \\
& 7.34 & 0.099 & 0.115 & 1.16 \\
& 14.8 & 0.099 & 0.110 & 1.16 \\
& 16.3 & 0.098 & 0.100 & 1.02 \\
& 17.3 & 0.099 & 0.147 & 1.50 \\
\hline
\end{tabular}




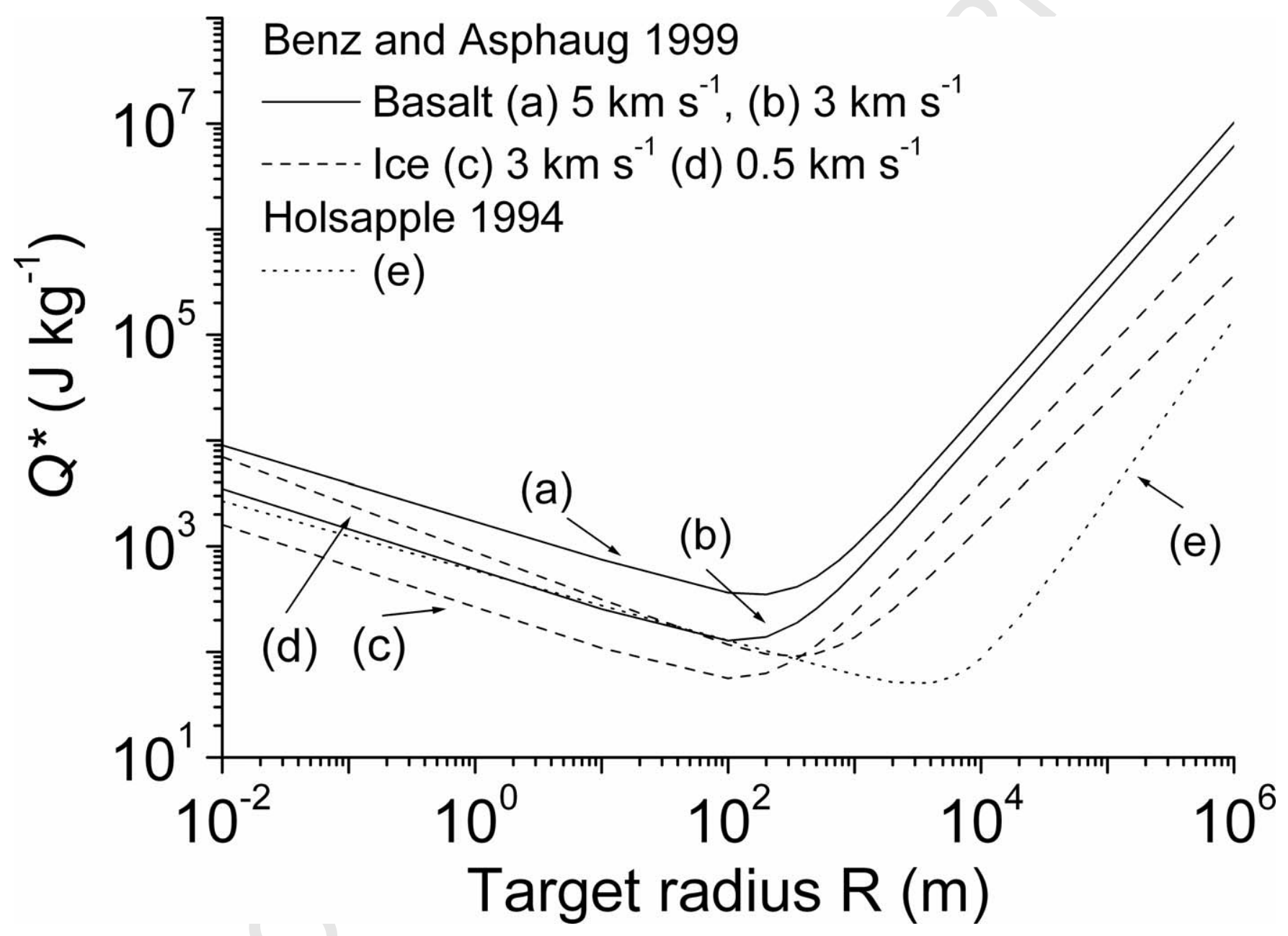




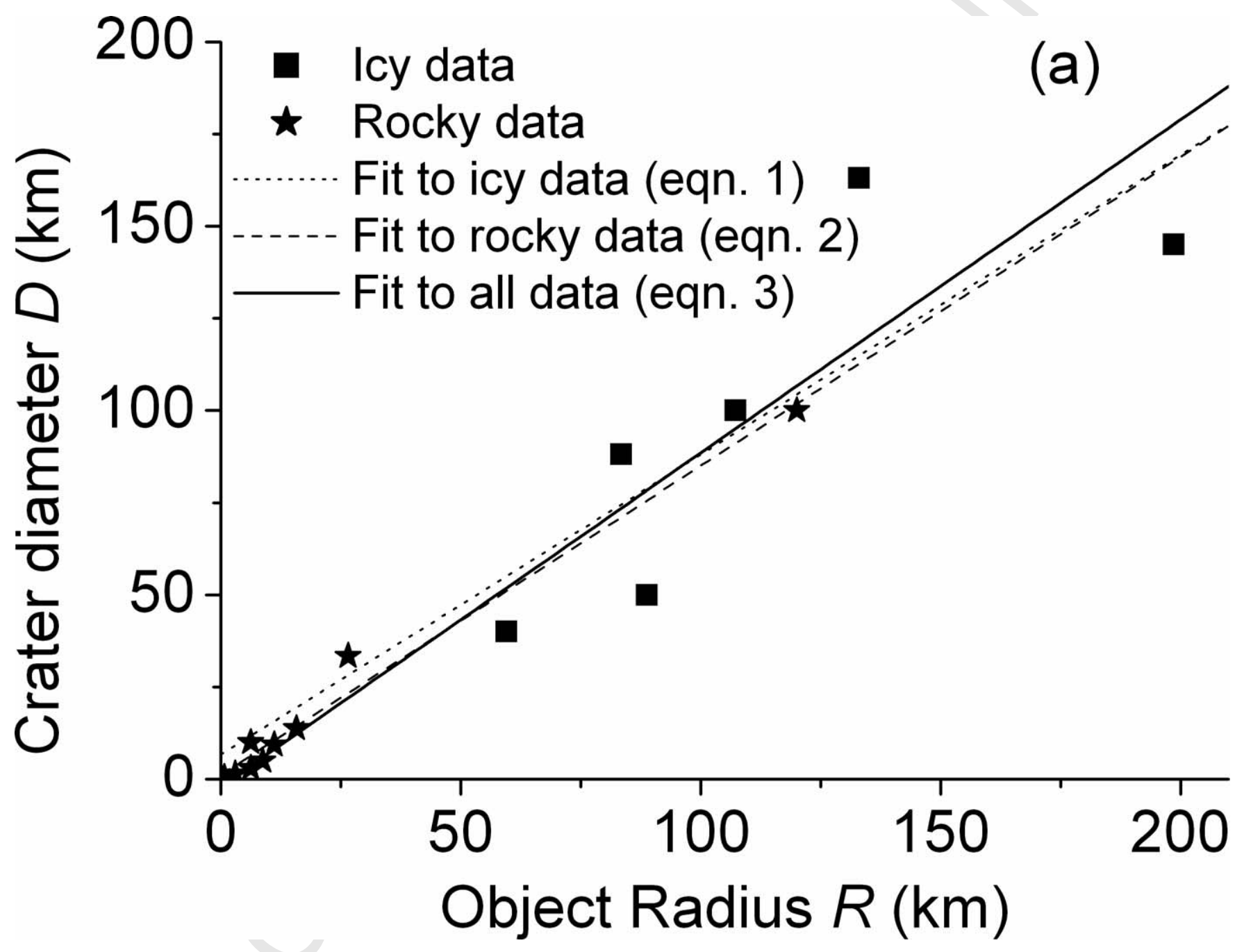




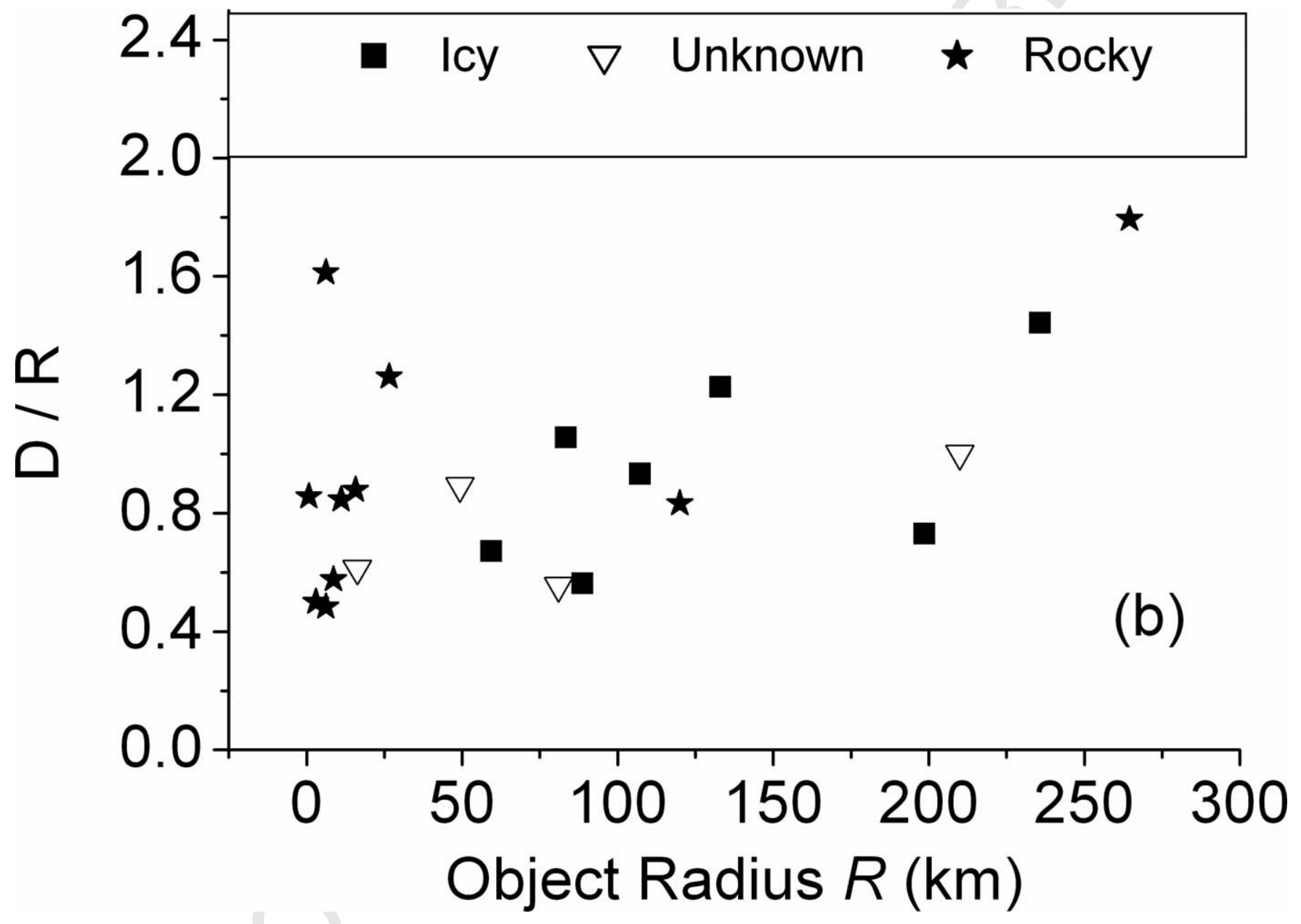




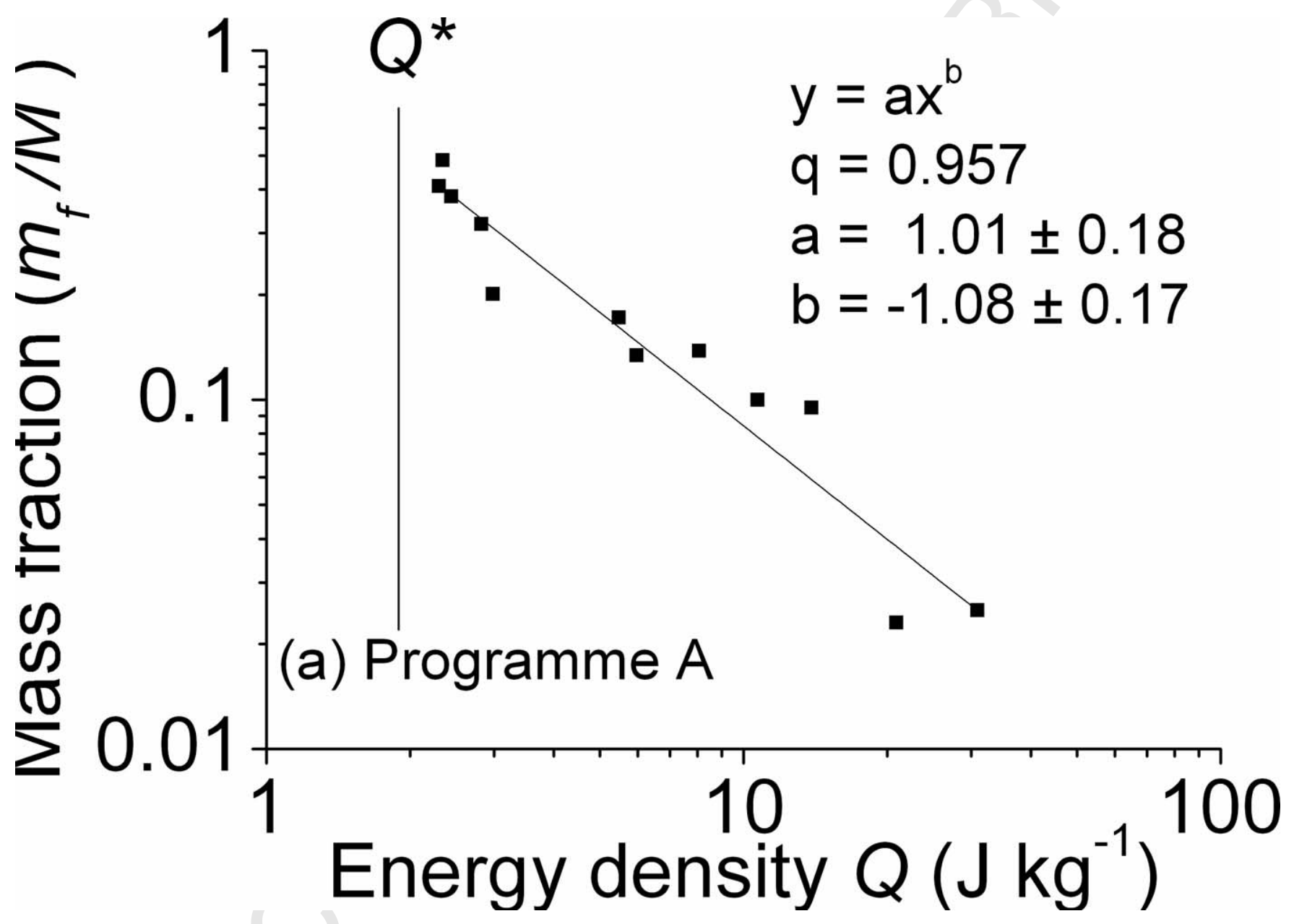




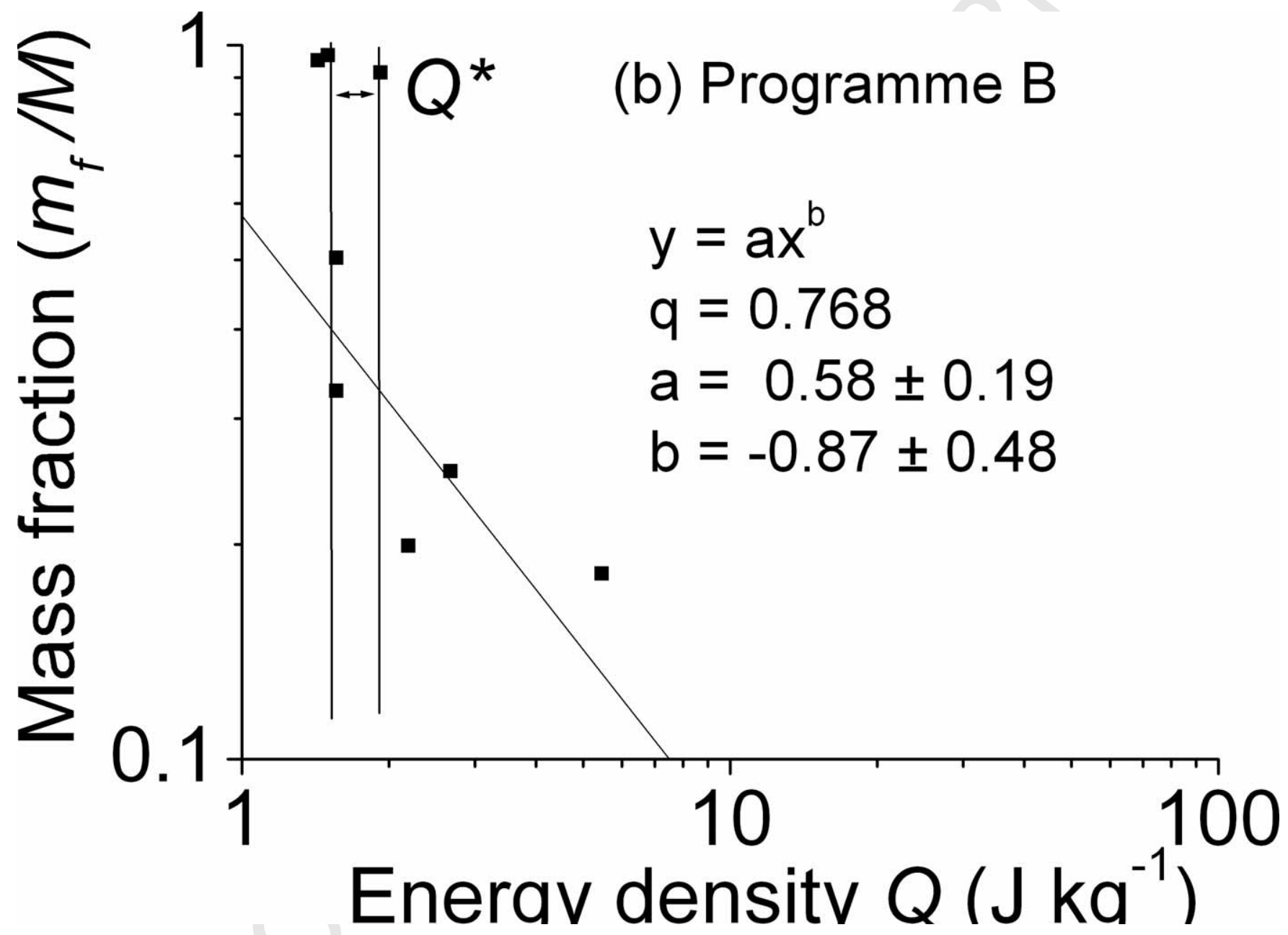




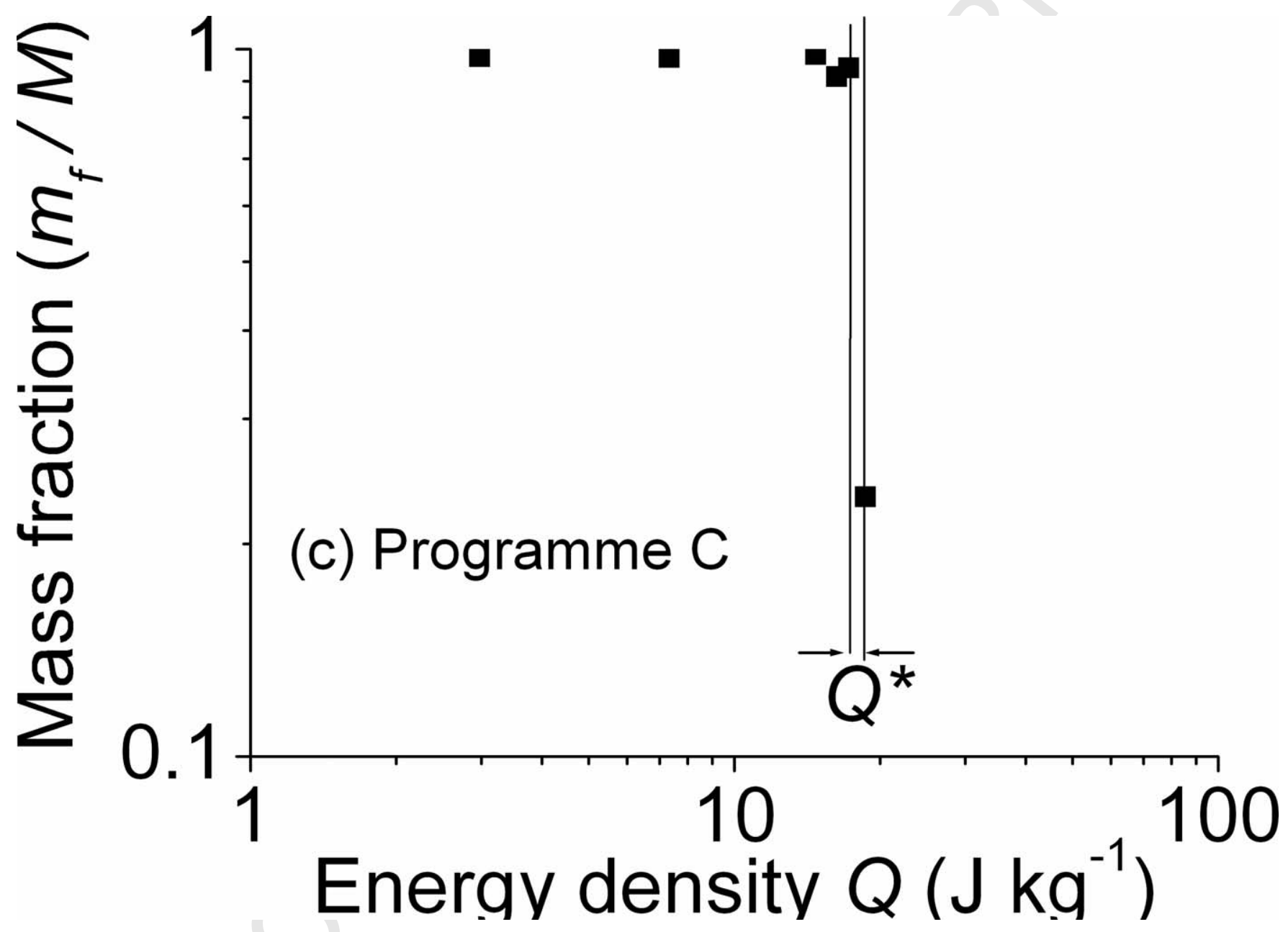




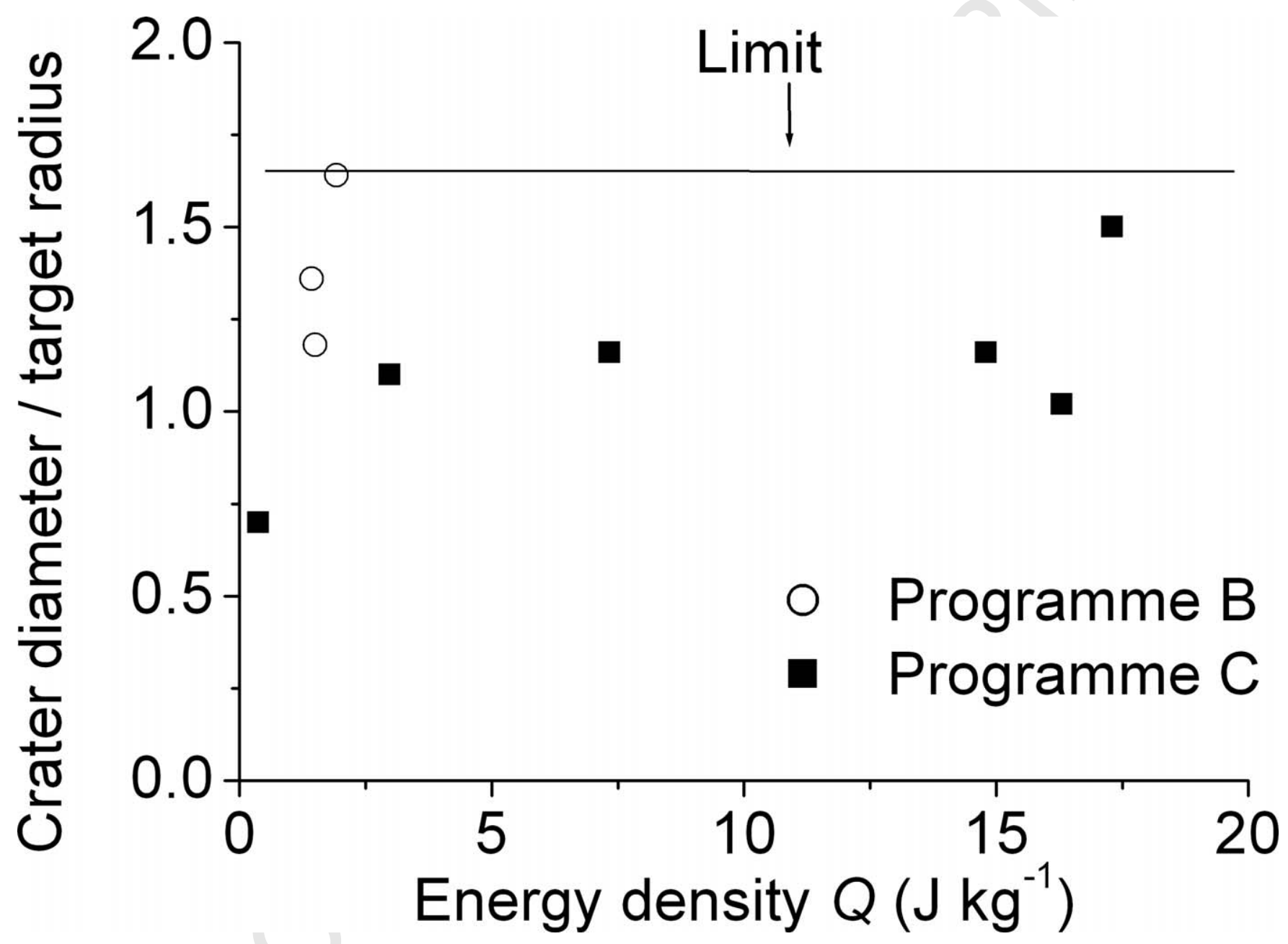




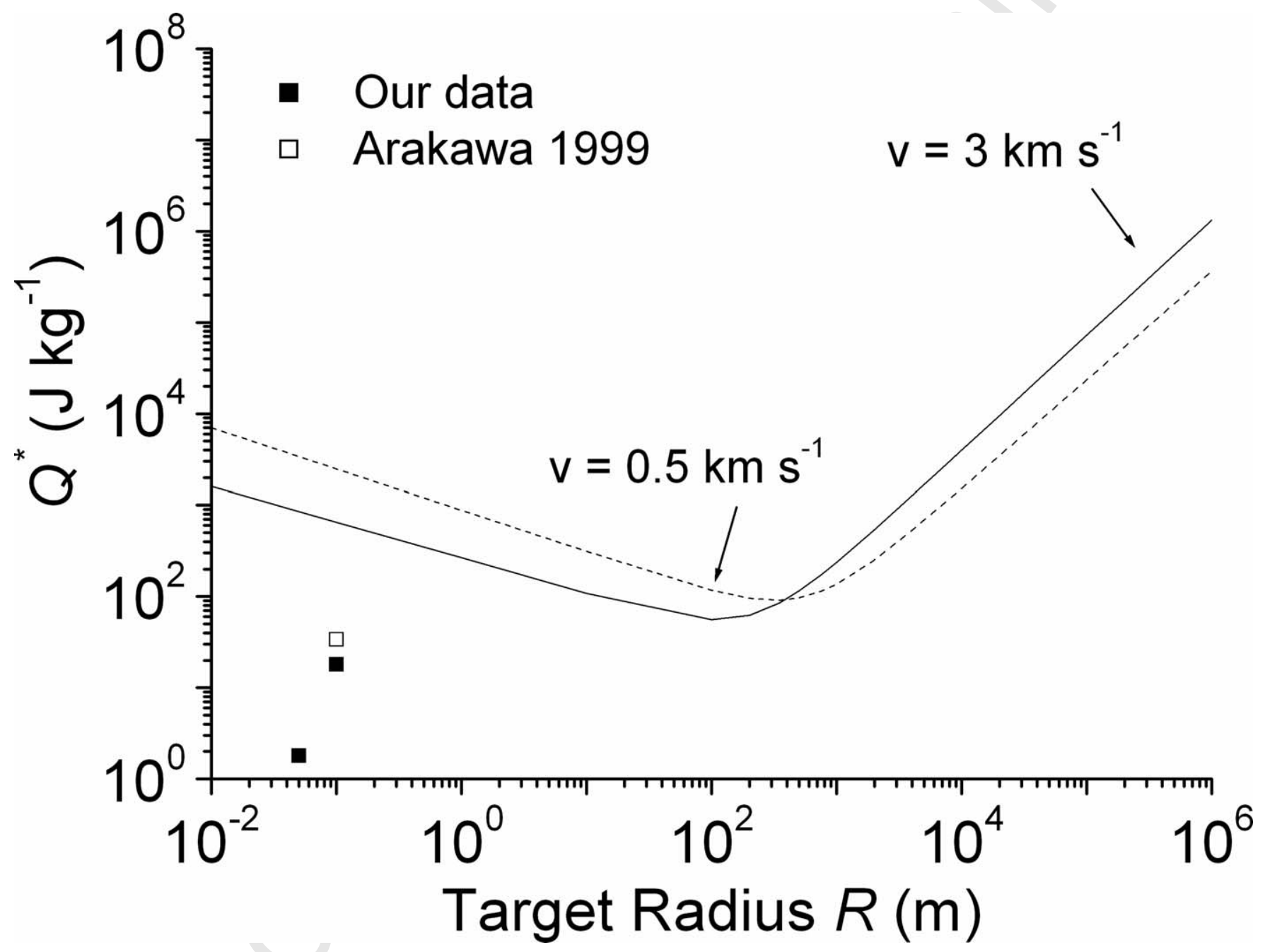


(a)

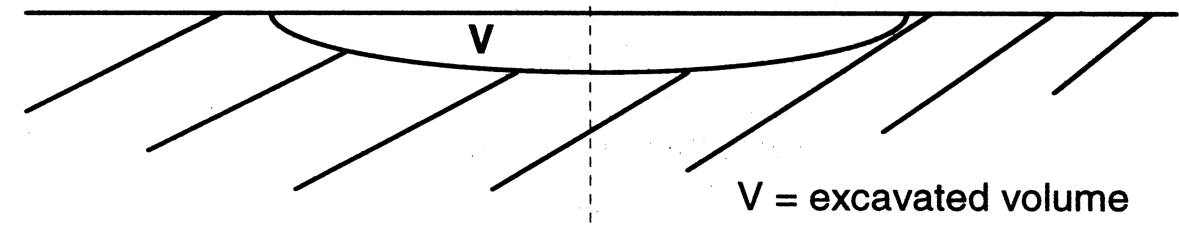

(b)

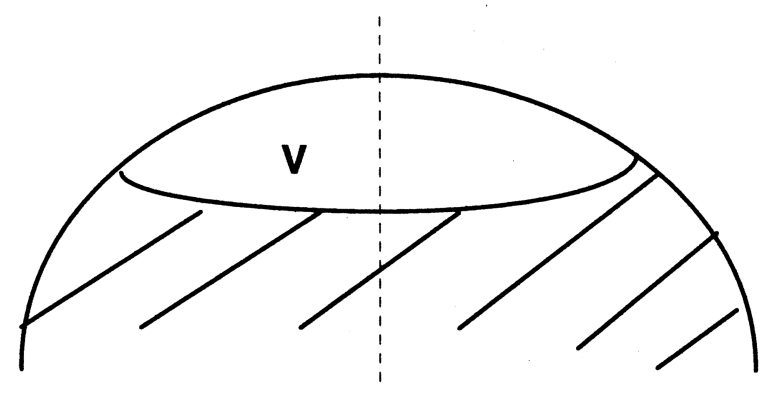

(c)

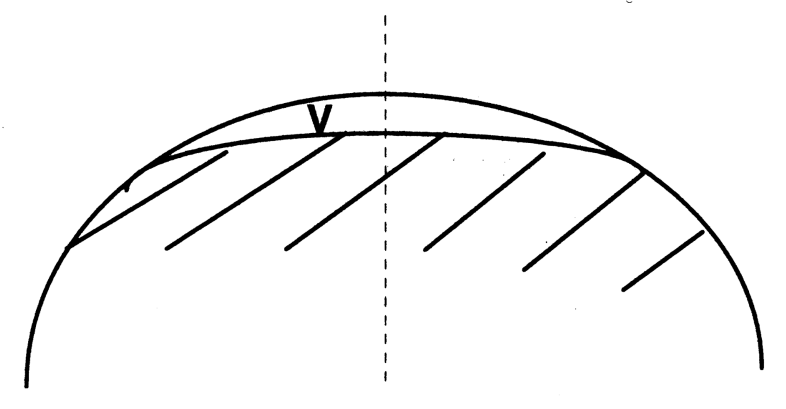

(d)

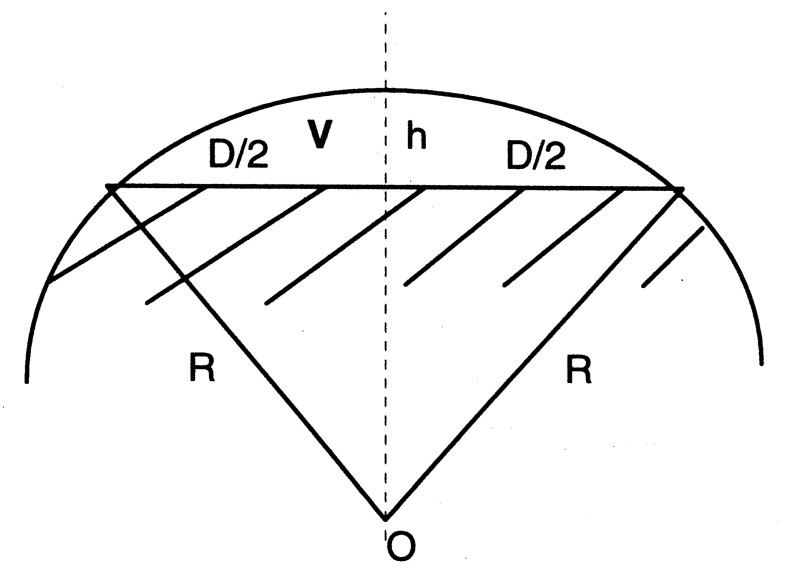

